\title{
Conductivity Maximum in 3D Graphene Foams
}

\author{
Feng Liu,* Chao Wang,* and Qiheng Tang
}

In conventional foams, electrical properties often play a secondary role. However, this scenario becomes different for 3D graphene foams (GrFs). In fact, one of the motivations for synthesizing 3D GrFs is to inherit the remarkable electrical properties of individual graphene sheets. Despite immense experimental efforts to study and improve the electrical properties of 3D GrFs, lack of theoretical studies and understanding limits further progress. The causes to this embarrassing situation are identified as the multiple freedoms introduced by graphene sheets and multiscale nature of this problem. In this article, combined with transport modeling and coarse-grained molecular dynamic (MD) simulations, a theoretical framework is established to systematically study the electrical conducting properties of 3D GrFs with or without deformation. In particular, through large-scale and massive calculations, a general relation between contact area and conductance for two van der Waals bonded graphene sheets is demonstrated, in terms of which the conductivity maximum phenomenon in GrFs is first theoretically proposed and its competition mechanism is explained. Moreover, the theoretical prediction is consistent with previous experimental observations.

In conventional foams, electrical property often plays a secondary role. However, this scenario becomes different for 3D GrFs. In fact, one of the motivations for synthesizing 3D GrFs is to inherit the remarkable electrical property of individual graphene sheet. Especially, much attention has been paid on electrical conductivity, since it decides the performance of $3 \mathrm{D} \mathrm{GrFs}$ on supercapacitors, flexible electronics, strain sensors, etc. Comparing with the direct density dependence in conventional foams, ${ }^{[31]}$ the decisive factors for electrical conductivity in 3D GrFs are not clear until now, despite a lot of measurements have been performed. As a matter of fact, the variation of electrical conductivity in 3D GrFs with respect to density has been recently summarized according to existing experimental data, and no direct link has been shown. ${ }^{[32]}$ This could be attributed to multiple freedoms introduced by graphene sheets, that is, their number of layers, characteristic length

\section{Introduction}

Graphene consists of 2D, one-atom-thick sheet of $\mathrm{sp}^{2}$-hybridized carbon atoms and has attracted tremendous interest in a variety of fields, due to its outstanding electronic, thermal, and mechanical properties. ${ }^{[1-4]}$ To tame these properties for applications in macroscale, both large-scale synthesis and integration of graphene sheets are indispensable. In the past few years, it witnesses the rapid development of synthesis technique in this field, 3D GrFs are now endowed with extreme properties encompassing superelasticity, ${ }^{[5-8]}$ ultralow density, ${ }^{[7]}$ high specific surface area, ${ }^{[9]}$ and good electrical conductivity. ${ }^{[8,10-15]}$ This could be the reason for their diverse potential applications, for example, energy storage and conversion, ${ }^{[16-19]}$ bioelectronics, ${ }^{[20-23]}$ strain sensors, ${ }^{[24]}$ supercapacitors electrodes, ${ }^{[25]}$ oil absorbers, ${ }^{[26-28]}$ and shock damping. ${ }^{[29,30]}$

Prof. F. Liu, Prof. C. Wang, Prof. Q. Tang

State Key Laboratory of Nonlinear Mechanics

Institute of Mechanics

Chinese Academy of Sciences

Beijing 100190, China

E-mail: liufeng@imech.ac.cn; wangchao@Inm.imech.ac.cn

Prof. Q. Tang

School of Engineering Science

University of Chinese Academy of Sciences

Beijing 100049, China

DOI: $10.1002 / \mathrm{smll} .201801458$ scale, and arrangement, which makes the fabrication of GrFs still dominated by a reverse engineering approach, that is, "aiming to study the properties of materials to be then able to build structures with improved mechanical and electrical characteristics by rational design." [32] To alleviate this situation, a theoretical study to figure out the decisive factors for electrical conductivity is in an urgent need.

Among various synthesis approaches, chemical vapor deposition method stands out as a successful way to obtain 3D GrFs with both high electrical conductivity and excellent mechanical properties. ${ }^{[15,33-37]}$ A common feature in these experiments is that compression could lead to the remarkable increase of electrical conductance suggesting the vital role of van der Waals contact between graphene sheets. Intuitively, this might originate from the rising contact area and contact nodes density between graphene sheets. However, to prove this point of view, both the microstructure of 3D GrFs and the conducting property of van der Waals bonded graphene sheets should be clarified. While the former could be figured out with coarse-grained MD simulations, to the best of our knowledge the latter has never been systematically studied, despite there are comprehensive transport studies about graphene nanoribbons. ${ }^{[38-40]}$

In this article, combining with the transport modeling as well as coarse-grained MD simulations, a theoretical framework has been established to systematically study the electrical conducting properties of 3D GrFs with or without deformation. First, large-scale and massive transport calculations, where the size of graphene sheets could reach to $125 \mathrm{~nm}$ (at least one 
order greater than the size of graphene nanoribbons normally used in transport theoretical studies), have been performed to demonstrate a general relation between contact area and conductance for two van der Waals bonded graphene sheets. Based on this result, the electrical conductivity of GrFs is then investigated, which is found to be closely related to the number of layers, especially there exists an optimum number of layers to obtain the conductivity maximum. As the classical optimization of foam density for thermal insulation is well known, ${ }^{[31]}$ which stems from the competition between the thermal conduction in solids and radiation to pass through solids, the existence of an optimum number of layers for the conductivity of 3D GrFs and the corresponding mechanism are first theoretically proposed and investigated, paving a way to optimize the conducting performance of 3D GrFs.

\section{Results and Discussion}

\subsection{Transport Modeling for Two van der Waals Bonded Graphene Sheets}

While a lot of theoretical studies have carefully studied the conductance of graphene nanoribbons, that for two van der Waals bonded graphene sheets has seldom been paid attention to. ${ }^{[41,42]}$ Especially, the influence of their size, twist angle, layer number, and contact area to the conductance is not clear. Since these factors are essential for understanding the conducting properties of GrFs, a transport model in this system needs to be established. To this end, a tight-binding (TB) model for multilayer graphene should be introduced first.

A TB model proposed in ref. [43] is used, and the total Hamiltonian is given by $H=H_{1}+H_{2}+H_{\text {int }}$, where $H_{1}$ and $H_{2}$ are Hamiltonians for the first and second layer of graphene, and $H_{\text {int }}$ represents the interaction between two layers (the details of this TB model are introduced in the Experimental Section). In Figure 1a, the configuration of AB-stacked bilayer graphene is shown with the top and side view of its unit cell in Figure 1b. Then, the corresponding band structure obtained in first principle calculations could be used to fit parameters in the TB model, and the comparisons between the TB model and first principle calculations are shown in Figure 1c, which is zoomed in Figure 1d. It can be seen that at low energy (less than $1.0 \mathrm{eV}$ deviation from Fermi level), the TB model could well describe the band structure. More importantly, the TB model is further validated when the twist angle is nonzero for the two van der Waals bonded graphene sheets, which could be found in the Supporting Information, as it will be used to consider the twist influence to the contact conductance.

With the TB model well established, the contact conductance could be systematically investigated. The top and side views of our transport model are shown in Figure 2a schematically, where different colors of atoms represent different layers. There are three regions in this model, that is, left lead region, conductor region, and right lead region. Note that a circular contact area is assumed and the hopping between the two layers is only turned on inside this contact area, which is marked in Figure 2a and used to consider the influence of the contact area to the conductance. In Figure 2b, the contact conductance for two van der Waals bonded graphene sheets with $30 \mathrm{~nm}$ in width and eight different contact areas is shown. With the energy deviating from Fermi level, a nearly linear increase in conductance could be clearly seen. Except the smaller magnitude, the energy dependence of conductance is similar to that of the monolayer graphene nanoribbon in the same width, which is marked with the pink solid line in Figure $2 \mathrm{~b}$. With voltages at two ends set to -1 and $1 \mathrm{~V}$, the corresponding variation of total conductance versus contact area is shown Figure 2c, and the conductance gradually reach its saturation as the size of contact area approaches the width of the graphene sheet. In experiments, 3D GrFs could be consisted by graphene sheets with different numbers of layers, and it is reported that the average number of layers could be controlled. ${ }^{[15]}$ Therefore, besides the size of the graphene sheets, the number of layers becomes another important factor to control the physical properties of 3D GrFs. Here, we simply extend the TB model of bilayer graphene for multilayer graphene, that is, $H=\sum_{i} H_{i}+\sum_{i, j} H_{\text {intij }}(i, j=1, \ldots, N$ and $|j-i|=1)$, so that the contact conductance of two van der Waals bonded graphene sheets with more than one layer, different twist angles, and different contact areas could be studied. Since graphene possesses both sixfold rotation and mirror symmetry, the twist angles ranging from $0^{\circ}$ to $30^{\circ}$ are sufficient to understand the twist influence to conductance. To be specific, the contact conductance for twist angles equaling $0^{\circ}, 6.59^{\circ}, 10.89^{\circ}$, $16.1^{\circ}, 23.41^{\circ}$, and $30^{\circ}$ are calculated and shown in Figure $2 \mathrm{~d}$, where each graphene sheet has three layers with ABA-stacking, and is $30 \mathrm{~nm}$ in width. A clear tendency is that with the twist angle becomes larger, the transport electrical gap enhances and the number of conducting channels decreases due to the aggravated lattice mismatch between the two graphene sheets. Note that in the real situation, the twist angle varies from different contact areas, and in fact they could even change in the same contact area. Therefore, the contact conductance for van der Waals bonded graphene sheets will be averaged with twist angles in the following.

\subsection{The Layer Number and Size Dependence of the Contact Conductance for Two van der Waals Bonded Graphene Sheets}

Next, our transport model is used to investigate the layer number and size dependence of the contact conductance for two van der Waals bonded graphene sheets. In principle, when the width of the graphene sheet is much larger than the carbon-carbon bond length, the size effect should be negligible. In this situation, given the same number of layers for graphene sheets, the evolution of normalized contact conductance $\bar{\sigma}=\sigma / \sigma_{d=w}$ with respect to the normalized contact area $\bar{S}=S / S_{d=w}=\left(\frac{\pi d^{2}}{4}\right) /\left(\frac{\pi w^{2}}{4}\right)$ should yield to one curve, where $d$ and $w$ represent the diameter of the circular contact area and the width of the graphene sheet, respectively.

To confirm this point of view, the contact conductance for graphene sheets with different widths and numbers of layers is calculated, and it is normalized with that of monolayer graphene sheets, that is, $\bar{\sigma}=\sigma / \sigma_{d=w, n=1}$, where $n$ is the number of layers. Indeed, it is found that the contact conductance for the graphene sheets with the same number of layers but 
(a)

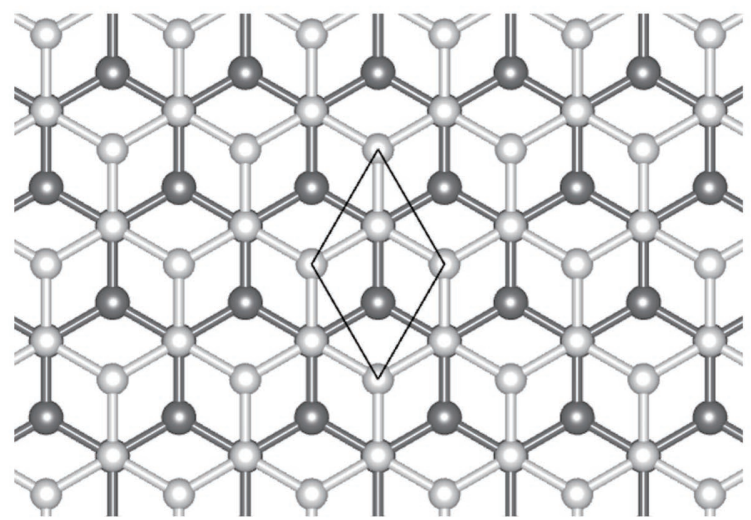

(b)

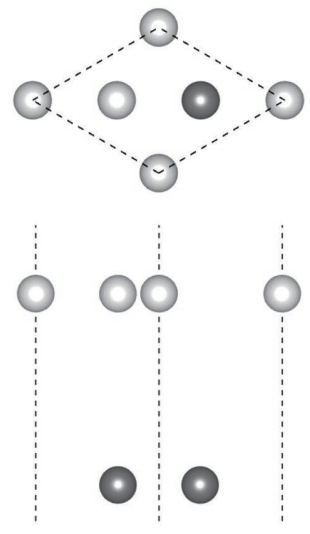

(c)

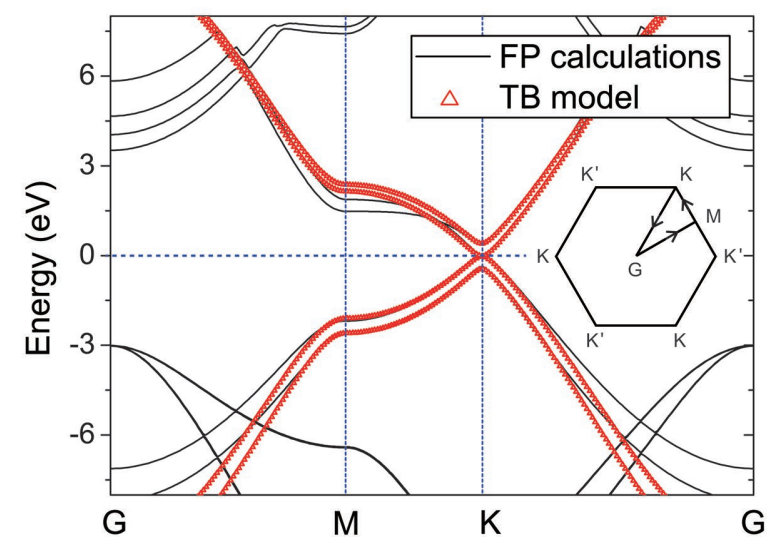

(d)

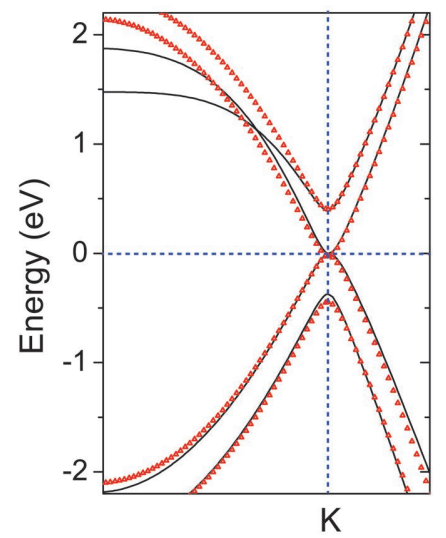

Figure 1. a) The configuration of AB-stacked bilayer graphene, and the unit cell used in our first principle calculation is shown. b) Top view (upper) and side view (lower) of the unit cell. c) The electronic band structure of bilayer graphene, where the dispersions from first principle calculations and TB model are marked with black solid line and red triangle, respectively. Inset: first Brillouin zone in the reciprocal space. d) Zoom in the dispersions at vicinity of $\mathrm{K}$ point.

different sizes shares the same change rule. Especially, when the contact conductance and area are normalized, they fall on one curve, which is consistent with the theoretical conjecture (see Figure 3a). However, with different numbers of layers, the rule may change. This could be understood physically, since with more layers for each graphene sheet the conducting efficiency slows down. For example, the contact conductance between bilayer graphene sheets is always less than two times of that between monolayer graphene sheets. To quantitatively describe the size dependence of contact conductance for different numbers of layers, the formula

$y=a x /(x+b)$

is used to fit the calculation results, where $a$ and $b$ are two fitting parameters. This formula gives two limits for the normalized conductance, that is, the lower limit (equals 0) and upper limit (approaches a constant $a$ ), which are corresponded to the noncontact and full-contact situations, respectively. Note that for different numbers of layers, the values of parameters can be different. The fitting results are plotted with solid lines in
Figure 3a, and different layers are marked with different colors. This fitting could well describe the calculation results, and the evolution of parameters with respect to the number of layers is shown in Figure $3 \mathrm{~b}$. It could be seen that as the number of layers increases, the values of both parameters increase suggesting the rising normalized conductance and the declined conducting efficiency. Note that the voltages at two ends are set to -1 and $1 \mathrm{~V}$, respectively, and this setting will be used in the following conductivity calculations unless otherwise stated. As complementary, different external voltages are tested and the change rule for the contact conductance is found to be similar (see the Supporting Information).

Besides, the variation of reference conductance, that is, the conductance for monolayer graphene sheets, with respect to the width of graphene sheets follows a power law as shown in Figure 3c. It provides a quantitative expression for the reference conductance, which combining with Equation (1) enables to predict the contact conductance for the van der Waals bonded graphene sheets with arbitrary width and number of layers.

One thing should be emphasized is that comparing with previous theoretical studies for the transport properties of 
(a)

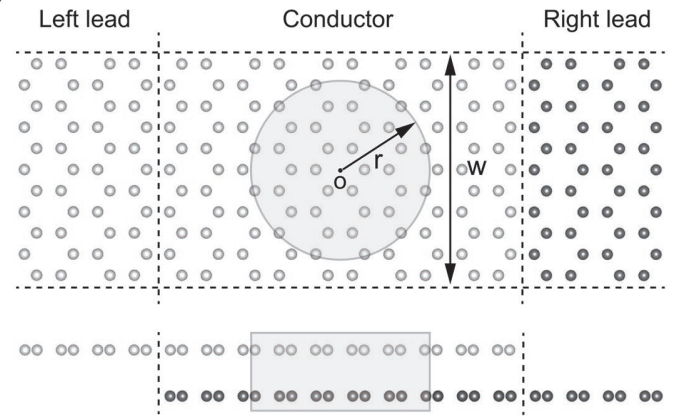

(b)

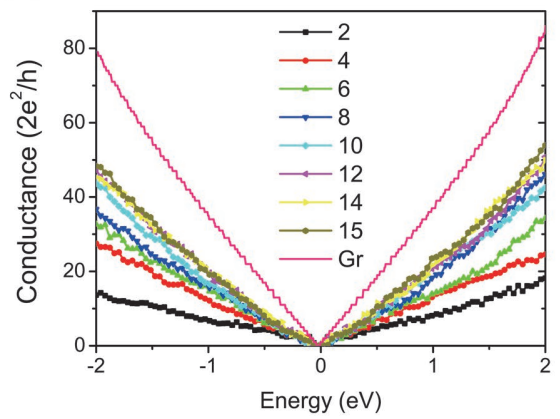

(c)

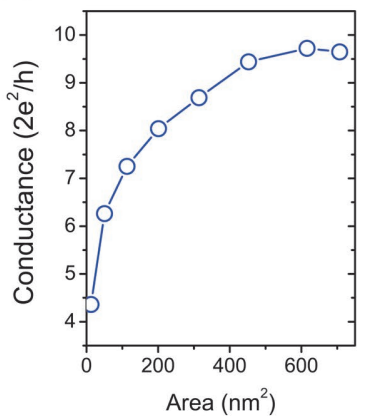

(d)

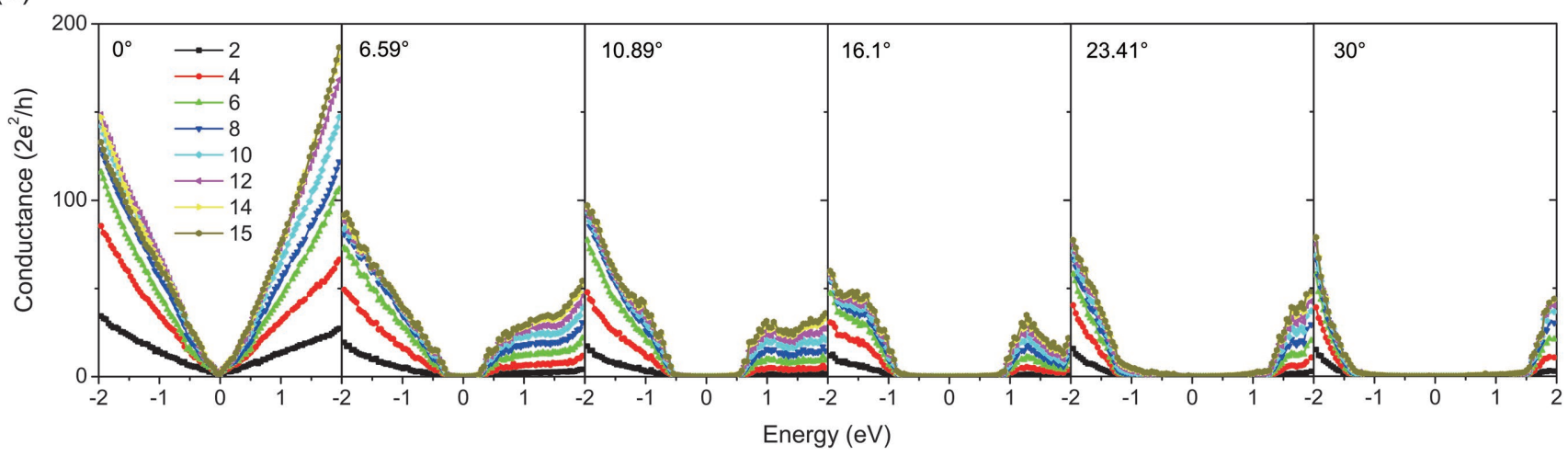

Figure 2. a) The schematic diagram of the mesoscopic transport model for AA-stacked van der Waals bonded graphene sheets. b) The energy dependence of contact conductance for two van der Waals bonded graphene sheets with $30 \mathrm{~nm}$ in width, where radiuses for contact areas equal 2, 4 , $6,8,10,12,14,15 \mathrm{~nm}$ and are marked with different colors. Note that the contact conductance of the $30 \mathrm{~nm}$ wide monolayer graphene nanoribbon is also given for comparison, which is marked with pink solid line. c) With voltages at two ends set to -1 and $1 \mathrm{~V}$, the variation of contact conductance versus contact area for AA-stacked van der Waals bonded graphene sheets. d) The evolution of the energy dependence of contact conductance for the van der Waals bonded ABA-stacked trilayer graphene sheets with six different twist angles, that is, $0^{\circ}, 6.59^{\circ}, 10.89^{\circ}, 16.1^{\circ}, 23.41^{\circ}$, and $30^{\circ}$.

graphene nanoribbons whose widths are usually limited to several nanometers, here large-scale and massive transport calculations have been performed to obtain Figure 3a, where the largest width for graphene sheets could reach to $125 \mathrm{~nm}$ and the largest layer number come up to eight. Multiple freedoms for graphene sheets, that is, the size, number of layers, twist angles, and contact area are considered in our calculations, and the cost for single calculation could be thousand times more than that for several nanometers wide graphene nanoribbons.

\subsection{The Conductivity Maximum Phenomenon in 3D GrFs}

The high conductivity is one major property pursued in $3 \mathrm{D}$ $\mathrm{GrFs}$, which is directly related to its functional applications. While a lot of experimental efforts have been devoted to measure and study the conductivity in GrFs, the theoretical studies and analyses are exceeding deficient or even nonexistent. As a result, its fundamental law is unclear and thus absent of effective optimization strategies. Next, the conductance of 3D GrFs and that of two van der Waals bonded graphene sheets will be correlated to form a theoretical framework, which, we believe, could help the researchers in this field get rid of the current awkward situation.

As mentioned above, the electron transport in 3D GrFs originates from the contact conductance of two van der Waals bonded graphene sheets. Physically, the 3D GrFs could be imaged as a network, where lines represent the individual graphene sheet, and nodes are on behalf of their contact regions. Therefore, the conductance of 3D GrFs is given by the contact conductance of the graphene sheets in series-parallel connection. A major concern here is whether the disordered distribution of graphene sheets in GrFs endows them with a novel conductivity behavior. To address this issue, a simple theoretical analysis is provided to compare the conductivity behavior in 3D GrFs with that for ordered stacked graphene sheets.

In Figure $4 \mathrm{a}$, the evolution of conductivities for triangular attached graphene sheets (upper inset in Figure 4a) and ordered stacked graphene sheets (lower inset in Figure 4a) with respect to number of layers is shown. Note that the triangular attached graphene sheets model is used to simulate the state of graphene sheets in GrFs, which are bended and attached with each other, and the corresponding conductivity is calculated by assuming a GrFs system possessing the same contact area per node and contact nodes density. With the enhanced number of layers, the structural and mechanical consequences are the increasing thickness and bending stiffness for graphene sheets. In ordered stacked graphene sheets, only the thickness of graphene sheets plays a role since no bending takes place, while for triangular attached graphene sheets the comparison between bending and adhesion energy determines the contact area, characteristic length scale, and thus conductivity. It could 
(a)
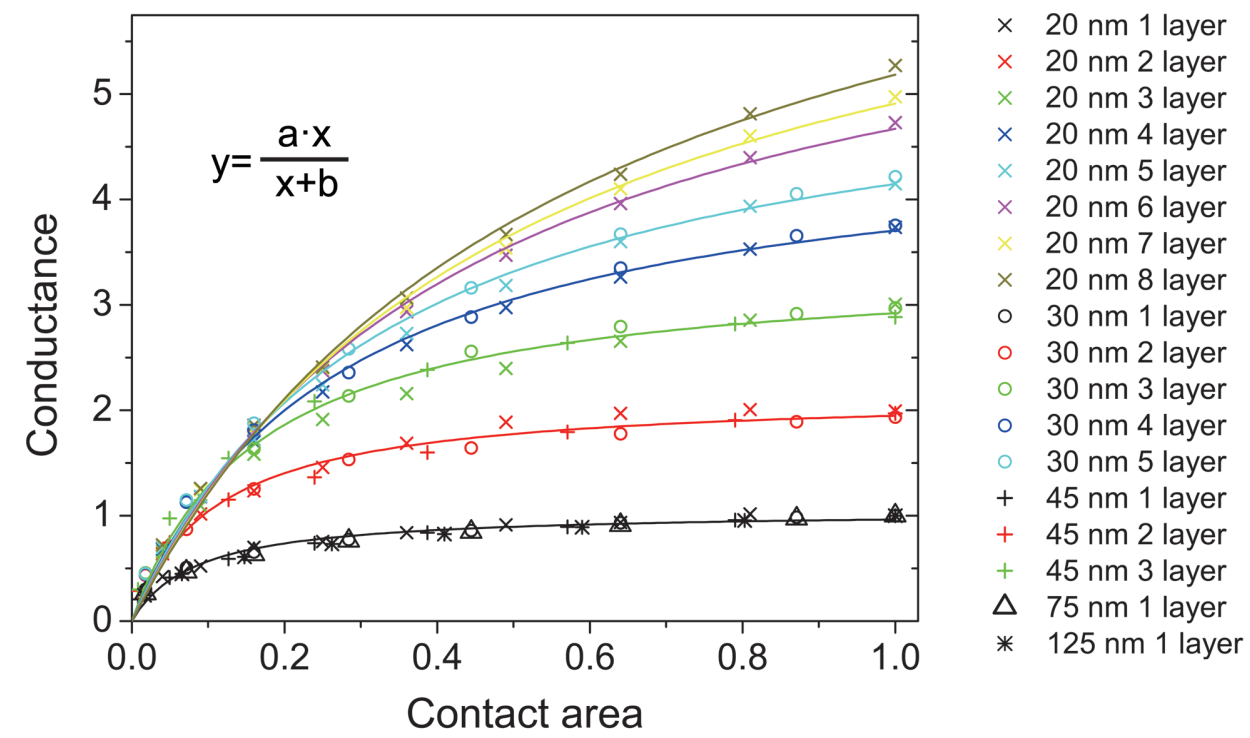

(b)

(c)
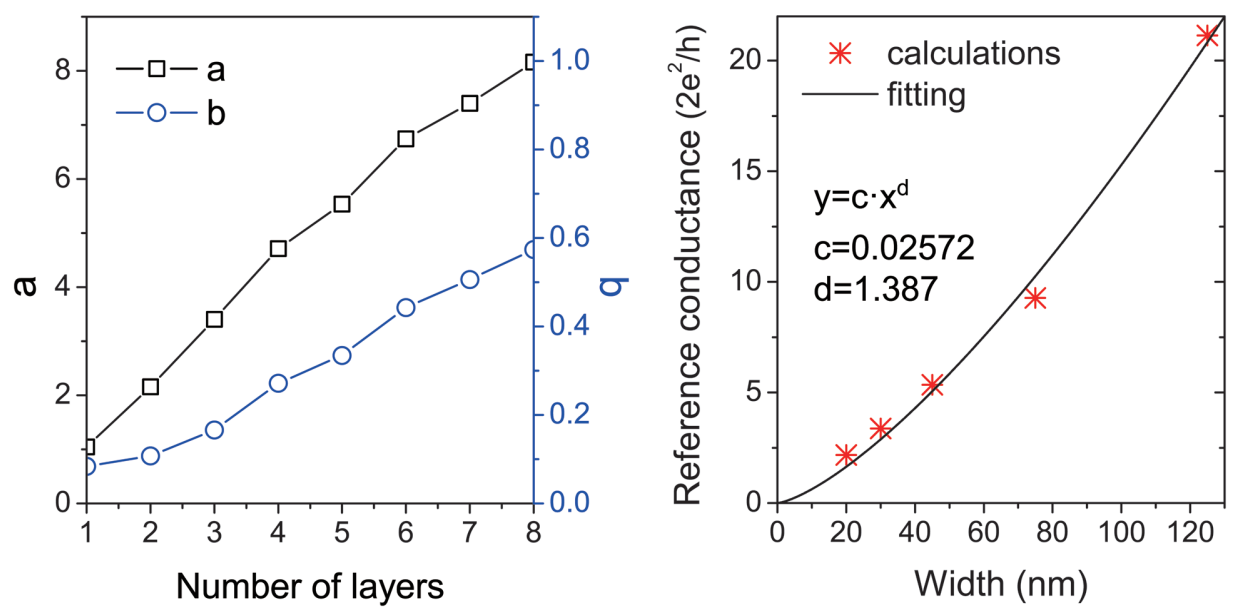

Figure 3. a) Normalized conductance with respect to normalized contact area, where Equation (1) is used to fit the calculation results and marked with solid lines. Note that graphene sheets with different widths are marked with different symbols, while graphene sheets with different numbers of layers are marked with different colors. b) The evolution of fitting parameters $a$ and $b$ with respect to number of layers. c) The reference conductance versus the width of graphene sheets, and it follows a power law.

be seen in Figure 4a that a conductivity maximum phenomenon emerges for triangular attached graphene sheets. However, this phenomenon is absent for ordered stacked graphene sheets. Note that the length and width of graphene sheets are set to $125 \mathrm{~nm}$, so that the theoretical result could be compared with the following calculations. More details about theoretical derivations and analyses could be found in the Supporting Information.

To figure out the essence of this difference, the competition mechanism should be clarified first. With the number of layers increase, there are three consequences, that is, the increasing bending stiffness, thickness for each graphene sheets, and contact conductance between them. The increasing bending stiffness could lead to the decrease in contact area per node (see Figure 4b), while the increasing thickness enhances the characteristic volume and thus lowers the contact nodes density (see Figure 4c). Despite in different ways, the first two consequences could reduce the conductivity, and the competition between the first two consequences and the third one could induce the conductivity maximum phenomenon. It is clear that if one of these consequences dominates, then the conductivity maximum phenomenon disappears. In fact, this is the case for the ordered stacked graphene sheets. In this system, nodes density is inversely proportional with the thickness of graphene sheets, and its declining rate is always larger than the growth rate of contact conductance between two van der Waals bonded graphene sheets, since the contact conductance for N-layer graphene sheets are always less than $\mathrm{N}$ times of its monolayer counterpart. As a result, the thickness variation for graphene sheets dominates the conductivity behavior and 
(a)

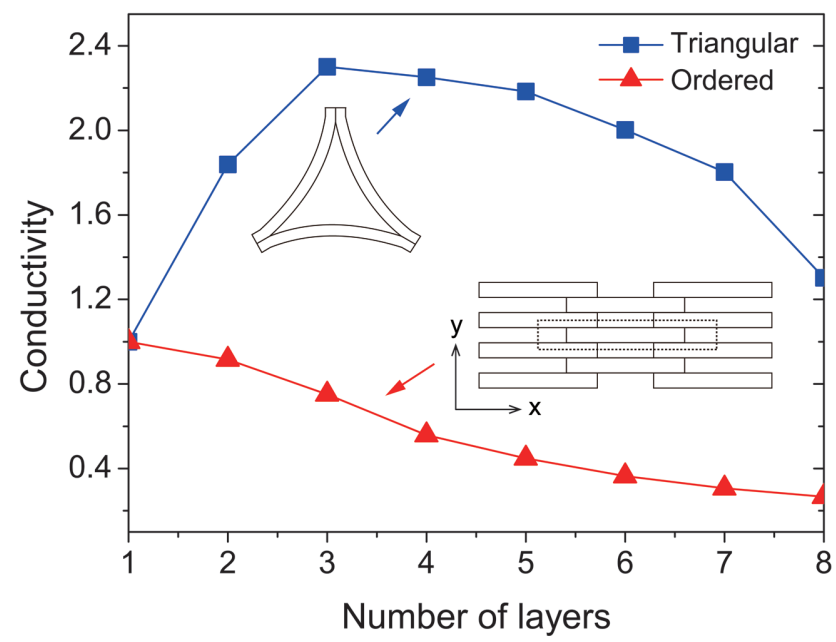

(b)

(c)
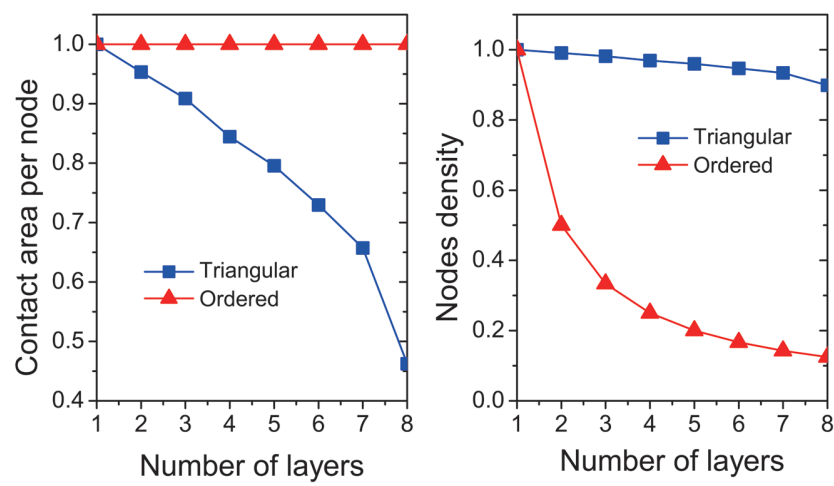

Figure 4. a) The variation of conductivity with respect to number of layers for two different systems, that is, triangular attached graphene sheets and ordered stacked graphene sheets. The insets show their configurations, which reflect the disordered and ordered stacked states for graphene sheets, respectively. Note that for ordered stacked graphene, $\sigma_{x x}$ is shown, and the conductivity is normalized with that of monolayer graphene sheets. The evolution of contact area per node and contact nodes density with respect to number of layers is shown in (b) and (c), and both of them are normalized similarly.

the conductivity maximum phenomenon disappears. In GrFs, the graphene sheets are distributed in a disordered manner, relative to thickness, the bending stiffness plays a much more important role with the increasing number of layers, and thus both the evolution of their structure and conductivity should be similar to the triangular attached graphene sheets model. It is expected that the conductivity maximum phenomenon should occur in GrFs.

To confirm this point of view, 3D GrFs are generated through MD simulations and their conductivity are directly calculated. For theoretical simplicity, two approximations are used in this process. First, it is assumed that all of the graphene sheets have the same number of layers $n$ and width $w$, which could be corresponded to the experimental average values. Second, the topological details for the connection of the network are neglected, instead two average quantities, that is, average contact area per node and nodes density, are used to predict the conductivity of 3D GrFs at a mean field level. With average contact area per node $S$ and nodes density $\rho$, the characteristic volume for one contact node $V=1 / \rho$, and the conductivity of 3D GrFs is given as

$\sigma=G(S, n, w) \cdot L / A=G(S, n, w) \cdot V^{-1 / 3}$

where $L$ and $A$ are characteristic length and area for one contact node, and $G(S, n, w)$ is the contact conductance of two graphene sheets and it is determined by $S, n$, and $w$. Note that the isotropic approximation is used in the final step. (The detailed illustration can be found in the Experimental Section, besides based on Equation (2) the theoretical maximum conductivity for given GrFs can be provided, see the Supporting Information)

Since the mesoscopic transport model could be used to obtain $G(S, n, w)$, now it only needs to obtain the structural information, that is, $S, n, w, \rho$, to calculate the conductivity of 3D GrFs by using Equation (2). To this end, the coarse-grained MD simulations are used to study the structure of 3D GrFs (the configuration generation and other simulation details could be found in the Experimental Section). In Figure 5a, a 3D GrFs sample with three layers and $125 \mathrm{~nm}$ in width is shown, where the contact regions are marked with red color, while the noncontact regions are marked with gray. In the left corner of Figure 5a, another color scheme is used for this sample, where different graphene sheets are plotted with different colors. The periodic boundary conditions are used and the size of the simulation box is about $460 \times 460 \times 460 \mathrm{~nm}^{3}$. In Figure $5 b, c$, the variation of contact area per node and nodes density with respect to the number of layers are shown with graphene sheets 75 and $125 \mathrm{~nm}$ in width. Comparing with the triangular attached graphene sheets model, the variation of contact area per node becomes less significant, and a much more dramatic decrease for the nodes density is observed. This may stem from the structure reconfiguration in GrFs samples due to the changed layer number of graphene sheets, which is not considered in the simple triangular attached graphene sheets model.

Despite there are some quantitative differences between the theoretical model and calculation results, the qualitative behavior remains, and thus the conductivity maximum phenomenon should take place. By using Equation (2), the evolution of conductivity versus the number of layers could be obtained and is shown in Figure 5d. The conductivity maximum occurs when the number of layers equals 3 , which is robust even when the width of graphene sheets is slightly changed (75 and $125 \mathrm{~nm}$ are shown in Figure 5d, 50 and $100 \mathrm{~nm}$ could be found in the Supporting Information). The reason is that with the normalized contact area around $10 \%$, the contact conductance shows a noticeable enhancement for graphene sheets within three layers, and is nearly unchanged for graphene sheets with more layers, at which time the continuous decreasing nodes density takes over to suppress the conductivity. It should be emphasized that the competition mechanism is irrelevant with the specific arrangement of graphene sheets, and thus the conductivity maximum phenomenon should be ubiquitous for 3D GrFs.

In fact, a lot of experiments have studied the conductivity behavior of $\mathrm{GrFs}^{[8,10-15]}$ and the existence of an optimum 
(a)

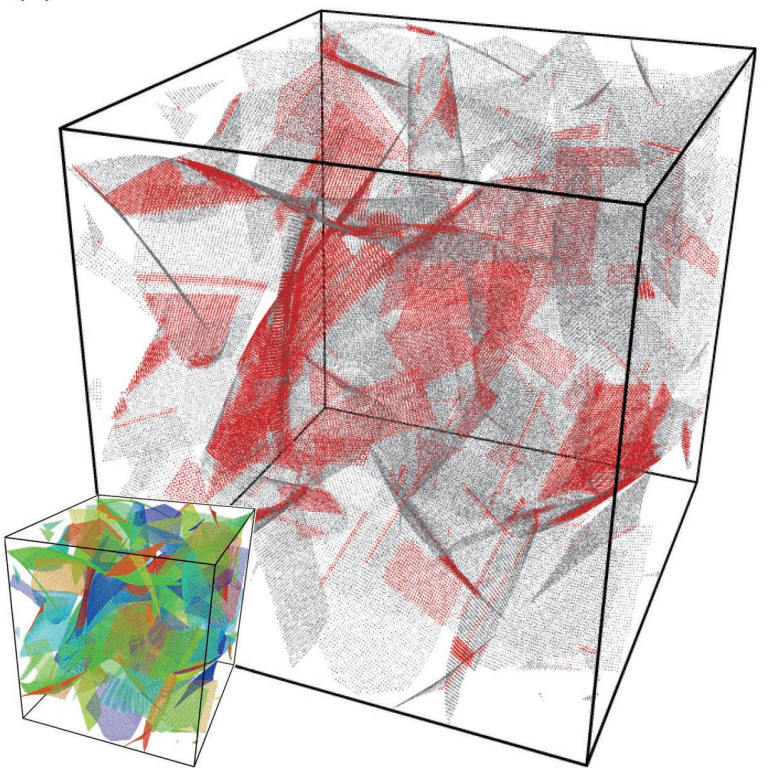

(b)

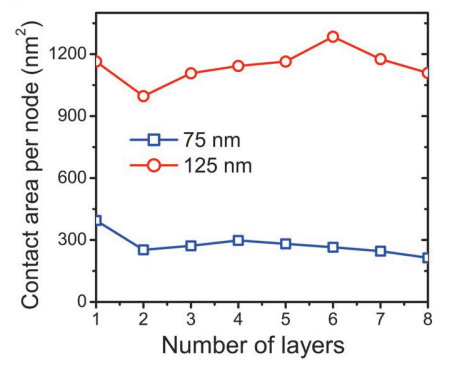

(d)

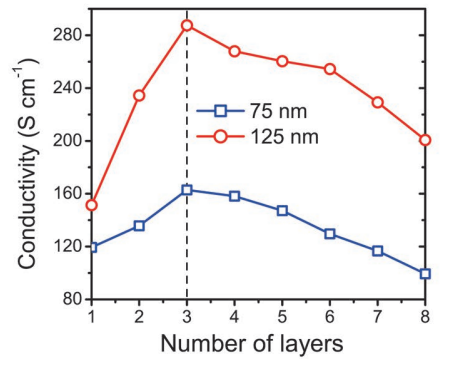

(c)

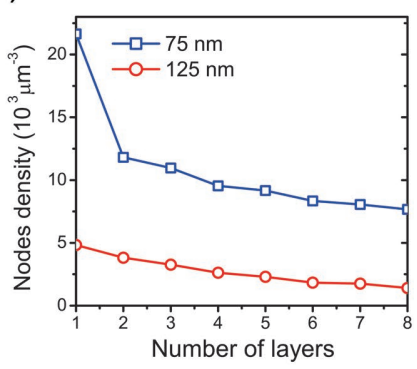

(e)

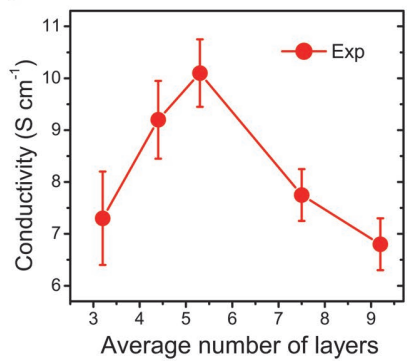

Figure 5. a) The snapshot of 3D GrFs, where graphene sheets have three layers and are $125 \mathrm{~nm}$ in width. The evolution of b) contact area per contact node, c) contact nodes density, and d) the corresponding conductivity with respect to number of layers. e) The variation of conductivity with respect to average number of layers according to the experimental measurements in ref. [15] is shown as comparison.

number of layers for the conductivity of 3D GrFs is recently reported, ${ }^{[15]}$ which is shown in Figure $5 e$ and the peak takes place at five layers. Despite a considerable difference in density for $3 \mathrm{D} \mathrm{GrFs}$, the conductivity maximum phenomenon is observed demonstrating the robustness of the competition mechanism. Since experimentally the graphene sheets in GrFs are usually at microscale, which are one order larger than the size of graphene sheets used in our simulations, the size effect in GrFs is carefully studied based on the triangular attached graphene sheets model and shown in the Supporting Information. It could be concluded that the adhesion energy takes a more important part relative to bending energy with the increasing size of graphene sheets, and it leads to a rise in the contact area per node, which in turn enhances the optimum number of layers for the conductivity maximum. This may possibly explain why the optimum number of layers in experiments is larger than its value obtained from our simulations. To the best of our knowledge, the conductivity maximum phenomenon in 3D GrFs and its competition mechanism are first theoretically proposed and investigated, based on which the conductivity of 3D GrFs could be optimized.

Until now, many different kinds of 3D $\mathrm{GrFs}^{[5,8,44-52]}$ are endowed with superelasticity, which combining with its wellconducting performance enables their potential applications in flexible electronics, strain sensors, bioelectronics, etc. For this reason, it is important to understand the conducting property of deformed 3D GrFs and to figure out its strain dependence. In the following, this issue will be studied based on our framework.

With coarse-grained MD simulations, the uniaxial tensile test is performed for a 3D GrFs sample composed by trilayer graphene sheets with $125 \mathrm{~nm}$ in width, and its microstructures at different strain levels are shown in Figure 6a. During the stretching process, it could be seen that the area and position of contact regions gradually evolve. Since in experiments the relative resistance is usually shown, its variation with respect to strain is given in Figure $6 \mathrm{~b}$, which presents an upward nonlinear increase. This is actually consistent with the measurements in recent experiments. ${ }^{[14,15]}$ Further, the cyclic loading is performed in our simulations. The variation of the relative resistance under the cyclic loading is shown in Figure 6c, where three tensile strain magnitudes, that is, 11, 32, and $54 \%$, are used. It is found the variation of resistance gradually becomes stable when the cyclic loading is more than five times, which is similar to the experimental observation. ${ }^{[8,10-15]}$ Especially, the relative resistance for the sixth circle (tensile strain magnitude equals $54 \%$ ) with respect to the strain is plotted in Figure 6d, and a slight hysteretic loop is present which also appears in experiments. ${ }^{[15]}$

Until now, various kinds of 2D materials have been successfully synthesized, which are accompanied by remarkable electrical, thermal, optical, and mechanical properties. 3D foam assembly serving as an effective, low-cost, and facile method to utilize these properties for macroscopic applications now becomes a new tendency. As the theoretical framework established here in principle could be used to study the conducting-related properties for 2D materials based 3D foams, it may provide theoretical supports for this novel direction.

In fact, the conducting performance of graphene foam is dominated by contact area. If we consider the electrical states near defects are localized or some adsorbates exist between graphene sheets, the general influence is to reduce the effective contact area. Thus, for different defects or adsorbates, we need to find the reduction of the effective contact area each defect, based on which we could predict the electric conductivity. Besides, when the concentration is up to some degree, the 
(a)

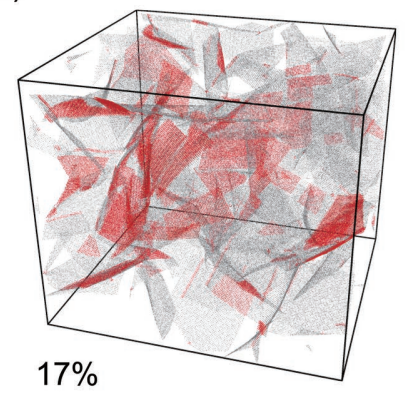

(b)

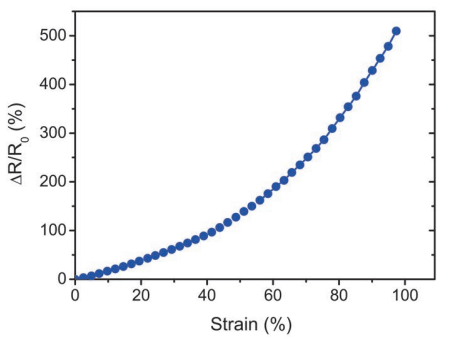

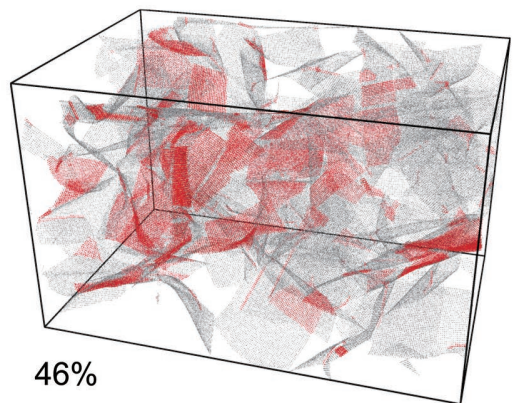

(c)

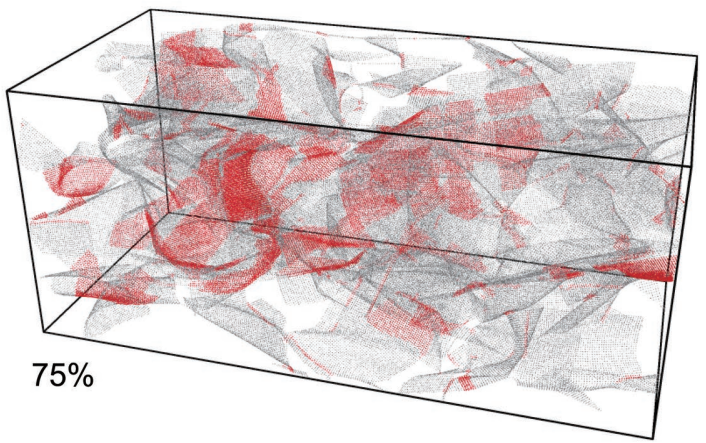

(d)
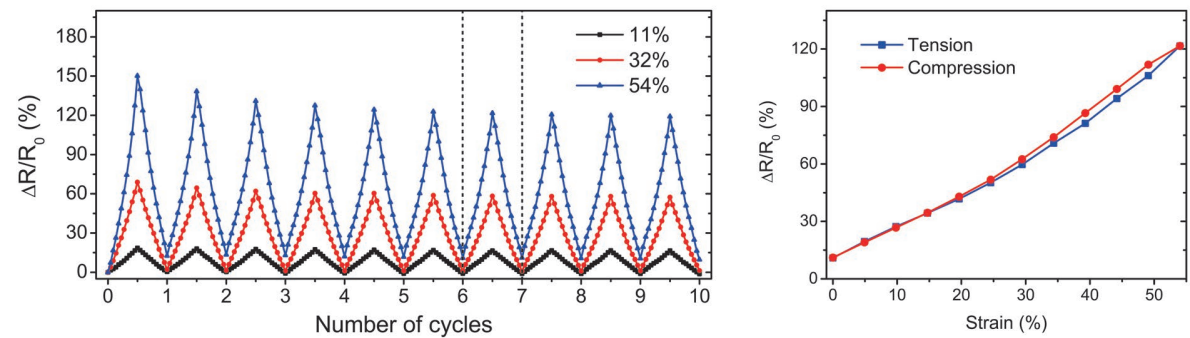

Figure 6. a) The structural evolution of 3D GrFs under stretching, where graphene sheets have three layers and are $125 \mathrm{~nm}$ in width. Three snapshots corresponding to different tensile strain levels, that is, 17, 46\%, and 75\%, are shown. b) The strain dependence of relative resistance. c) The variation of relative resistance with respect to the number of loading cycles, and three different magnitudes of tensile strain are used and marked with different colors. d) The variation of relative resistance with strain for the sixth cycle, where the tensile strain magnitude equals $54 \%$.

doping effect should be taken into account, Fermi level could deviate from zero in this case. Therefore, the defect effect could be an interesting topic in our further study.

\section{Conclusion}

Combining with the transport modeling as well as coarsegrained MD simulations, we establish a theoretical framework, based on which, the electrical conducting property of 3D GrFs is systematically investigated. Particularly, a fundamental correlation between contact conductance and contact area for two van der Waals bonded graphene sheets is uncovered, which serves as the cornerstone of our theoretical framework. Since this correlation has nothing to do with the exact arrangement of the graphene sheets, our theoretical framework is universal for 3D GrFs with arbitrary configuration, and in principle it could be extended to study the conducting property for other 2D materials based foams.

By taking advantage of this framework, an interesting theoretical prediction is that there exists an optimum number of layers to obtain conductivity maximum in GrFs. The competition mechanism stems from the contact conductance enhancement between graphene sheets while a continuous decrease for the nodes density as the number of layers for graphene sheets increase, and it gives us much confidence to learn that our theoretical prediction is just consistent with the previous experimental observation. Besides, the strain-dependent conduction for 3D GrFs is investigated, and the primary experimental features are reproduced, which once again validate our theoretical framework.

\section{Experimental Section}

Variables Illustration: Equation (2) is the most important equation in the theoretical framework, which correlates the conductivity of GrFs with the contact conductance of two graphene sheets G. Here, the related variables used in this equation are illustrated carefully.

Characteristic volume for one contact node $V=1 / \rho$, which represents the average volume for one contact node. Based on the isotropic approximation (the structure is uniform and does not depend on direction), the characteristic length and area could be given as $V^{1 / 3}$ and $V^{2 / 3}$, respectively, which leads to the simplified form of Equation (2), that is, $\sigma=G(S, n, w) \cdot V^{-1 / 3}$. Note that $\rho$ is nodes density, and it could be obtained in the simulations by counting the number of contact nodes inside the simulation box. Besides, $G(S, n, w)$ is the contact conductance of two graphene sheets determined by average contact area per node $S$, the number of layers $n$, and the width of the graphene sheet $w$ (see Figure 3 ).

3D Numerical Sample of GrFs: The coarse-grained scheme of multilayer graphene sheet proposed by Cranford and Buehler ${ }^{[33]}$ is adopted here. A square coarse-grained flake was used as a building block. All flakes were placed randomly in a cubic box with periodic boundary condition in three directions. Under the Berendsen barostat 1 barometric pressure and Langevin thermostat $300 \mathrm{~K}$, the system reaches the final equilibrium state at about $10 \mathrm{~ns}$ with the criterion that the total energy fluctuation converges to less than $1 \%$. With some spring bonds connecting the neighboring flakes, the ten cyclic uniform tension-compression simulations were adopted to study the variation of microstructural contact of the system. All simulations were implemented using an open source software Large-scale Atomic/Molecular Massively Parallel Simulator (LAMMPS) ${ }^{[54]}$ with a time step $10 \mathrm{fs}$. The open visualization tool (OVITO) ${ }^{[55]}$ was used to generate configurational illustrations. More details could be found in the Supporting Information.

First Principle Calculations Details: The geometrical configurations and electric band structures of bilayer graphene were obtained selfconsistently by using the projector augmented wave pseudopotential method implemented in the VASP package. ${ }^{[56]}$ The energy cutoff and 
convergence criteria are set to be $400 \mathrm{eV}$ and $10^{-5} \mathrm{eV}$, respectively, and the maximum allowed force on each atom for structure optimization is less than $10^{-3} \mathrm{eV} \AA^{-1}$. Besides, the Perdew-Burke-Ernzerhof (PBE) exchangecorrelation potential[ ${ }^{[5]}$ was adopted for electronic structure calculations.

TB Model: A TB model proposed in ref. [43] was used to study the conductivity of two van der Waals bonded graphene sheets, and the Hamiltonian is given by $H=H_{1}+H_{2}+H_{\text {int }}$, where $H_{1}$ and $H_{2}$ are Hamiltonians for the first and second layer of graphene according to Reich model, ${ }^{[58]}$ and $H_{\text {int }}=\gamma_{\mathrm{t}} e^{-\left|r_{i j}-d_{i j}\right| / \beta}$ represents the interaction between two layers. Here, $r$ is the vector pointing from the ith atom to the $j$ th atom, $d$ is a vector with its magnitude equalling the interlayer distance and its direction pointing from the graphene layer including ith atom to another graphene layer including $j$ th atom, and $\gamma_{t}$ and $\beta$ are hopping parameter and characteristic distance, respectively. The fitting values of parameters in Reich model are $\varepsilon_{2 \mathrm{p}}=0.927 \mathrm{eV}, \gamma_{0}=-3.105 \mathrm{eV}$, $s_{0}=0 \mathrm{eV}, \gamma_{1}=0.023 \mathrm{eV}, s_{1}=0 \mathrm{eV}, \gamma_{2}=0.273 \mathrm{eV}, s_{2}=0 \mathrm{eV}$, the definition of which could be found in ref. [58] and those in $H_{\text {int }}$ are $\gamma_{t}=0.769 \mathrm{eV}$ and $\beta=0.423 \AA$. Note that the zero overlap matrix elements, that is, $s_{1}=0$ and $s_{2}=0$, are assumed to facilitate calculations of the transport model. This model could be extended for multilayer graphene, that is, $H=\sum_{i} H_{i}+\sum_{i, j} H_{\text {int } ; j, j}$, where $i, j=1, \ldots, N$ and $|j-i|=1$.

Note that at low energy (less than $1.0 \mathrm{eV}$ deviation from Fermi level), the TB model could well describe the band structure and thus give an accurate prediction for the conducting property.

Recursion Green's Function Method: As the system is quite large, the recursion Green's function method ${ }^{[59]}$ was used to obtain the contact conductance of two van der Waals bonded graphene sheets. The advanced Green's function of the conductor region is written as

$G_{\mathrm{C}}=\lim _{\eta \rightarrow 0^{+}}\left[(E+i \eta) I-H_{\mathrm{C}}-\Sigma_{\mathrm{L}}-\Sigma_{\mathrm{R}}\right]^{-1}$

where $\Sigma_{\mathrm{L}}$ and $\Sigma_{\mathrm{R}}$ are self-energies of left and right leads, respectively, and $H_{C}$ represents the Hamiltonian of the conductor region. The conductance is given as

$G(E)=\frac{2 e^{2}}{h} \operatorname{Tr}\left(\Gamma_{\mathrm{R}} G_{\mathrm{C}} \Gamma_{\mathrm{L}} G_{\mathrm{C}}^{\dagger}\right)$

where $\Gamma_{L, R}=i\left[\Sigma_{L, R}-\Sigma_{L, R}^{*}\right]$ are broadening matrices. The total conductance is written as

$G=\frac{1}{\mu_{2}-\mu_{1}} \int_{\mu_{1}}^{\mu_{2}} G(E) d E$

where $\mu_{1}$ and $\mu_{2}$ are the voltages at two ends.

\section{Supporting Information}

Supporting Information is available from the Wiley Online Library or from the author.

\section{Acknowledgements}

The research was supported by the National Natural Science Foundation of China (Grant Nos. 11602272, 11602270, 11790292, 11021262, 11172303, 11132011, and 11532013), the National Basic Research Program of China ("973" Project) (Grant No. 2012CB937500), and the Strategic Priority Research Program of the Chinese Academy of Sciences (Grant No. XDB22040503)

\section{Conflict of Interest}

The authors declare no conflict of interest.

\section{Keywords}

3D graphene foams, conductivity, first principle, MD simulations, strain

Received: April 17, 2018

Revised: May 26, 2018

Published online:

[1] A. H. Castro Neto, N. M. R. Peres, K. S. Novoselov, A. K. Geim, Rev. Mod. Phys. 2009, 81, 109.

[2] K. S. Novoselov, A. K. Geim, S. V. Morozov, D. Jiang, M. I. Katsnelson, I. V. Grigorieva, S. V. Dubonos, A. A. Firsov, Nature 2005, 438, 197.

[3] A. A. Balandin, S. Ghosh, W. Z. Bao, I. Calizo, D. Teweldebrhan, F. Miao, C. N. Lau, Nano Lett. 2008, 8, 902.

[4] F. Bonaccorso, Z. Sun, T. Hasan, A. C. Ferrari, Nat. Photonics 2010, $4,611$.

[5] Y. P. Wu, N. B. Yi, L. Huang, T. F. Zhang, S. L. Fang, H. C. Chang, N. Li, J. Oh, J. A. Lee, M. Kozlov, A. C. Chipara, H. Terrones, P. Xiao, G. K. Long, Y. Huang, F. Zhang, L. Zhang, X. Lepro, C. Haines, M. D. Lima, N. P. Lopez, L. P. Rajukumar, A. L. Elias, S. M. Feng, S. J. Kim, N. T. Narayanan, P. M. Ajayan, M. Terrones, A. Aliev, P. F. Chu, Z. Zhang, R. H. Baughman, Y. S. Chen, Nat. Commun. 2015, 6, 6141.

[6] X. Xu, Q. Q. Zhang, Y. K. Yu, W. L. Chen, H. Hu, H. Li, Adv. Mater. 2016, 28, 9223.

[7] L. Qiu, B. Huang, Z. J. He, Y. Y. Wang, Z. M. Tian, J. Z. Liu, K. Wang, J. C. Song, T. R. Gengenbach, D. Li, Adv. Mater. 2017, 29, 1701553.

[8] L. Qiu, J. Z. Liu, S. L. Chang, Y. Wu, D. Li, Nat. Commun. 2012, 3, 1241.

[9] Y. Qian, I. M. Ismail, A. Stein, Carbon 2014, 68, 221.

[10] C. Zhu, T. Y. Han, E. B. Duoss, A. M. Golobic, J. D. Kuntz, C. M. Spadaccini, M. A. Worsley, Nat. Commun. 2015, 6, 6962.

[11] Q. Zhang, F. Zhang, S. P. Medarametla, H. Li, C. Zhou, D. Lin, Small 2016, 12, 1702

[12] B. H. Min, D. W. Kim, K. H. Kim, H. O. Choi, S. W. Jang, H.-T. Jung, Carbon 2014, 80, 446.

[13] M. A. Worsley, P. J. Pauzauskie, T. Y. Olson, J. Biener, J. H. Satcher, T. F. Baumann, J. Am. Chem. Soc. 2010, 132, 14067.

[14] X. M. Li, T. T. Yang, Y. Yang, J. Zhu, L. Li, F. E. Alam, X. Li, K. L. Wang, H. Y. Cheng, C. T. Lin, Y. Fang, H. W. Zhu, Adv. Funct. Mater. 2016, 26, 1322.

[15] Z. P. Chen, W. C. Ren, L. B. Gao, B. L. Liu, S. F. Pei, H. M. Cheng, Nat. Mater. 2011, 10, 424

[16] B. G. Choi, M. Yang, W. H. Hong, J. W. Choi, Y. S. Huh, ACS Nano 2012, 6, 4020.

[17] L. L. Jiang, L. Z. Sheng, C. L. Long, T. Wei, Z. J. Fan, Adv. Energy Mater. 2015, 5, 1500771.

[18] C. G. Hu, L. Song, Z. P. Zhang, N. Chen, Z. H. Feng, L. T. Qu, Energy Environ. Sci. 2015, 8, 31.

[19] J. S. Lee, H. J. Ahn, J. C. Yoon, J. H. Jang, Phys. Chem. Chem. Phys. 2012, 14, 7938

[20] J. Y. Liu, X. H. Wang, T. S. Wang, D. Li, F. N. Xi, J. Wang, E. K. Wang, ACS Appl. Mater. Interfaces 2014, 6, 19997.

[21] A. E. Jakus, E. B. Secor, A. L. Rutz, S. W. Jordan, M. C. Hersam, R. N. Shah, ACS Nano 2015, 9, 4636.

[22] P. C. Sherrell, B. C. Thompson, J. K. Wassei, A. A. Gelmi, M. J. Higgins, R. B. Kaner, G. G. Wallace, Adv. Funct. Mater. 2014, 24, 769.

[23] E. Murray, S. Sayyar, B. C. Thompson, R. Gorkin, D. L. Officer, G. G. Wallace, RSC Adv. 2015, 5, 45284

[24] J. Kuang, L. Q. Liu, Y. Gao, D. Zhou, Z. Chen, B. H. Han, Z. Zhang, Nanoscale 2013, 5, 12171. 
[25] Y. X. Xu, K. X. Sheng, C. Li, G. Q. Shi, ACS Nano 2010, 4, 4324.

[26] H. Sun, Z. Xu, C. Gao, Adv. Mater. 2013, 25, 2554.

[27] S. Barg, F. M. Perez, N. Ni, P. do Vale Pereira, R. C. Maher, E. Garcia-Tunon, S. Eslava, S. Agnoli, C. Mattevi, E. Saiz, Nat. Commun. 2014, 5, 4328.

[28] H. C. Bi, X. Xie, K. B. Yin, Y. L. Zhou, S. Wan, L. B. He, F. Xu, F. Banhart, L. T. Sun, R. S. Ruoff, Adv. Funct. Mater. 2012, 22, 4421.

[29] H. Hu, Z. B. Zhao, W. B. Wan, Y. Gogotsi, J. S. Qiu, Adv. Mater. 2013, 25, 2219.

[30] A. L. R. Bug, S. A. Safran, G. S. Grest, I. Webman, Phys. Rev. Lett. 1985, 55, 1896

[31] L. J. Gibson, M. F. Ashby, Cellular Solids: Structure and Properties, Cambridge University Press, Cambridge 1999.

[32] P. C. Sherrell, C. Mattevi, Mater. Today 2016, 19, 428.

[33] R. Munoz, C. Gomez-Aleixandre, Chem. Vap. Deposition 2013, 19297.

[34] Y. Zhang, L. Y. Zhang, C. W. Zhou, Acc. Chem. Res. 2013, 46, 2329.

[35] A. Reina, X. T. Jia, J. Ho, D. Nezich, H. B. Son, V. Bulovic, M. S. Dresselhaus, J. Kong, Nano Lett. 2009, 9, 30.

[36] X. S. Li, W. W. Cai, L. Colombo, R. S. Ruoff, Nano Lett. 2009, 9, 4268.

[37] Y. Zhang, L. Gomez, F. N. Ishikawa, A. Madaria, K. Ryu, C. A. Wang, A. Badmaev, C. W. Zhou, J. Phys. Chem. Lett. 2010, 1, 3101.

[38] M. Y. Han, B. Ozyilmaz, Y. B. Zhang, P. Kim, Phys. Rev. Lett. 2007, 98, 206805.

[39] C. Gomez-Navarro, R. T. Weitz, A. M. Bittner, M. Scolari, A. Mews, M. Burghard, K. Kern, Nano Lett. 2007, 7, 3499.

[40] S. Das Sarma, S. Adam, E. Hwang, E. Rossi, Rev. Mod. Phys. 2011, $83,407$.

[41] J. W. Gonzalez, H. Santos, M. Pacheco, L. Chico, L. Brey, Phys. Rev. B 2010, 81, 195406.

[42] K. M. M. Habib, F. Zahid, R. K. Lake, Appl. Phys. Lett. 2011, 98, 192112.
[43] E. S. Morell, J. D. Correa, P. Vargas, M. Pacheco, Z. Barticevic, Phys. Rev. B 2010, 82, 121407.

[44] Y. Tao, X. Y. Xie, W. Lv, D. M. Tang, D. B. Kong, Z. H. Huang, H. Nishihara, T. Ishii, B. H. Li, D. Golberg, F. Y. Kang, T. Kyotani, Q. H. Yang, Sci. Rep. 2013, 3, 2975.

[45] Q. G. Shao, J. Tang, Y. X. Lin, F. F. Zhang, J. S. Yuan, H. Zhang, N. Shinya, L. C. Qin, J. Mater. Chem. A 2013, 1, 15423.

[46] S. M. Yoon, W. M. Choi, H. Baik, H. J. Shin, I. Song, M. S. Kwon, J. J. Bae, H. Kim, Y. H. Lee, J. Y. Choi, ACS Nano 2012, 6, 6803.

[47] Y. Yuan, L. Y. Liu, M. L. Yang, T. L. Zhang, F. Xu, Z. S. Lin, Y. J. Ding, C. H. Wang, J. J. Li, W. L. Yin, Q. Y. Peng, X. D. He, Y. B. Li, Carbon 2017, 123, 223.

[48] Y. Zhao, C. G. Hu, L. Song, L. X. Wang, G. Q. Shi, L. M. Dai, L. T. Qu, Energy Environ. Sci. 2014, 7, 1913.

[49] H. Bi, I. W. Chen, T. Q. Lin, F. Q. Huang, Adv. Mater. 2015, 27, 5943.

[50] Y. C. Wang, Y. B. Zhu, F. C. Wang, X. Y. Liu, H. A. Wu, Carbon 2017, $118,588$.

[51] Z. Li, Z. Xu, Y. J. Liu, R. Wang, C. Gao, Nat. Commun. 2016, 7, 13684.

[52] L. Z. Sheng, T. Wei, Y. Liang, L. L. Jiang, L. T. Qu, Z. J. Fan, Carbon 2017, 120, 17.

[53] S. Cranford, M. J. Buehler, Modell. Simul. Mater. Sci. Eng. 2011, 19, 054003.

[54] S. Plimpton, J. Comput. Phys. 1995, 117, 1.

[55] A. Stukowski, Modell. Simul. Mater. Sci. Eng. 2010, 18, 015012.

[56] G. Kresse, J. Furthmuller, Phys. Rev. B 1996, 54, 11169.

[57] J. P. Perdew, K. Burke, M. Ernzerhof, Phys. Rev. Lett. 1996, 77, 3865.

[58] S. Reich, J. Maultzsch, C. Thomsen, P. Ordejon, Phys. Rev. B 2002, 66, 035412 .

[59] F. Teichert, A. Zienert, J. Schuster, M. Schreiber, J. Comput. Phys. 2017, 334, 607. 